\title{
Neutral Inhomogeneity in Circular Cylinder Subjected to Axial Load on its Lateral Boundary
}

\author{
I. ECSEDI ${ }^{1}$, Á. J. LENGYEL ${ }^{2}$, A. BAKSA ${ }^{3}$ \\ ${ }^{1}$ University of Miskolc, Faculty of Mechanical Emgineering and Informatics, Institute of Applied Mechanics, \\ mechecs@uni-miskolc.hu \\ ${ }^{2}$ University of Miskolc, Faculty of Mechanical Emgineering and Informatics, Institute of Applied Mechanics, \\ mechlen@uni-miskolc.hu \\ ${ }^{3}$ University of Miskolc, Faculty of Mechanical Emgineering and Informatics, Institute of Applied Mechanics, \\ mechab@uni-miskolc.hu
}

Abstract. In this paper we consider the problem of single circular elastic inhomogeneity embedded within a circular cylinder whose curved boundary surface is subjected to surface traction acting on axial direction. We investigate the displacement neutrality of the coupled system of host body and inclusion. Neutral inhomogeneity (inclusion) does not disturb the displacement, strain and stress fields in the host body. The deformation of the considered inhomogenneous cylinder is antiplane shear deformation.

Keywords: circular cylinder, elastic inclusion, neutral inhomogeneity, antiplane shear deformation

\section{Introduction}

The antiplane shear deformation is a special case of the state of deformation in a solid body. This state is achived when the displacements in the body are zero in the plane of interest but nonzero in the direction perpendicular to the plane. If the plane of antiplane shear deformation is the plane Oxy of the rectangular Cartesian frame $O x y z$ and the displacement vector is represented as

$$
\mathbf{u}=u \mathbf{e}_{x}+v \mathbf{e}_{y}+w \mathbf{e}_{z},
$$

where $\mathbf{e}_{x}, \mathbf{e}_{y}, \mathbf{e}_{z}$ are unit vectors in $x, y$ and $z$ directions, then the antiplane shear deformation is defined by the next equations $[1,2,3]$

$$
u=0, \quad v=0, \quad w=w(x, y) .
$$

This means that if we consider a cylindrical body (Fig. 1) whose generators are parallel to axis $z$, all cross sections of this body have same deformations according to Eq. (2). The strain field of infinitesimal antiplane shear deformation is expressed as

$$
\gamma_{x z}=\frac{\partial w}{\partial x}, \quad \gamma_{y z}=\frac{\partial w}{\partial y}
$$

where $\gamma_{x z}=\gamma_{x z}(x, y)$ and $\gamma_{y z}=\gamma_{y z}(x, y)$ are the shearing strains, other strains are zero. The cross section of the cylindrical body is a simply connected bounded plane domain, it is denoted by $A$. The 
boundary curve of $A$ is indicated by $\partial A$ (Fig. 1). In our example $A$ is a solid circular domain (Fig. 2). It is assumed that the material of the considered host body is homogeneous cylindrical orthotropic linearly elastic. The material of the cylindrical inclusion is isotropic, homogeneous and linearly elastic. The coupled system of host body and inclusion is shown in Fig. 2. The radius of cylindrical boundary surface of circular inclusion is $a$, and the radius of circular boundary of host body is $R$ (Fig. 2). The connection between the host body and inclusion is perfect. The coupled system of host body and inclusion is called $z$-homogeneous cylindrical bar since the material parameters depend only on the cross sectional coordinates $x, y$. In this paper we consider the problem of a single circular elastic inclusion embedded within a circular cylinder whose boundary surface is loaded by axial traction. We investigate the displacement neutrality of the coupled host body and inclusion. The netural inhomogeneity does not disturb the displacement, strain and stress fields in the host body.

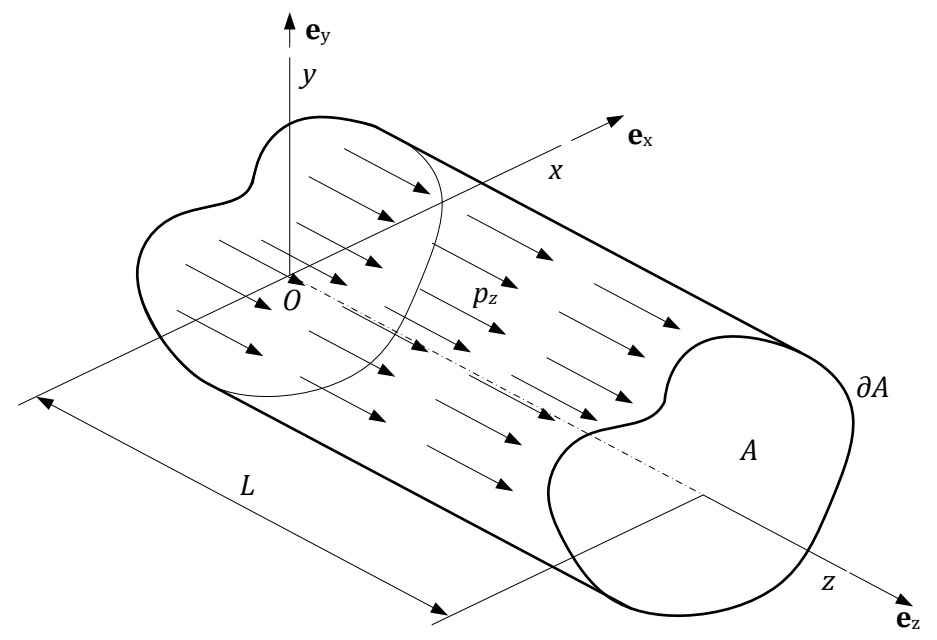

Figure 1. Cylindrical body with equilibrium surface traction.
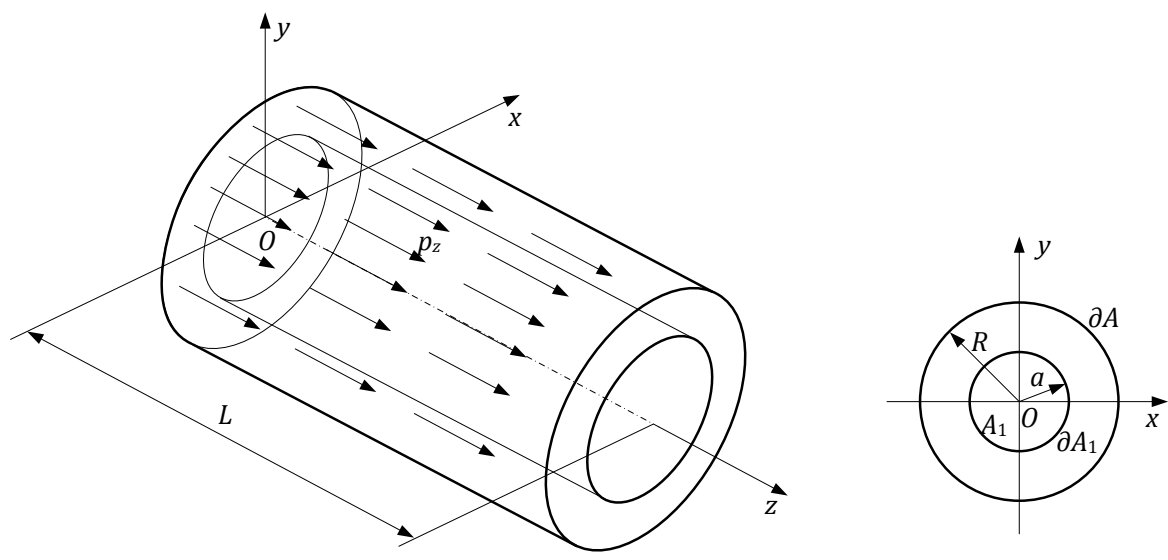

Figure 2. Circular cylinder with inclusion.

\section{Governing equations for host cylinder}

To formulate the governing equations the cylindrical polar coordinate system $\operatorname{Or} \varphi z$ will be used. The connection of Cartesian coordinates $x, y$ and cylindrical coordinates $r, \varphi$ is as follows 
International Journal of Engineering and Management Sciences (IJEMS) Vol. 5. (2020). No. 2

DOI: 10.21791/IJEMS.2020.2.5.

$$
x=r \cos \varphi, \quad y=r \sin \varphi .
$$

Denote the axial displacement in the host body $w_{0}=w_{0}(r, \varphi)$. The strain and stress field in the cylindrical orthotropic body in antiplane shear deformation can be computed as

$$
\begin{array}{cc}
\gamma_{r z}=\frac{\partial w_{0}}{\partial r}, & \gamma_{\varphi z}=\frac{1}{r} \frac{\partial w_{0}}{\partial \varphi}, \\
\tau_{r Z}=G_{r z} \gamma_{r Z}, & \tau_{\varphi z}=G_{\varphi z} \gamma_{\varphi Z},
\end{array}
$$

where $G_{r z}$ and $G_{\varphi z}$ are the shear moduli of cylindrical orthotropic elastic material, $\tau_{r z}$ and $\tau_{\varphi z}$ are the shearing stresses. The stress equilibrium equation in present problem $[1,3]$

$$
\frac{\partial \tau_{r Z}}{\partial r}+\frac{\tau_{r Z}}{r}+\frac{1}{r} \frac{\partial \tau_{\varphi Z}}{\partial \varphi}=0, \quad(r, \varphi) \in A
$$

Combination of Eqs. (5) and (6) with Eq. (7) gives the next equation for the axial displacement $w_{0}=w_{0}(r, \varphi)$

$$
\frac{\partial^{2} w_{0}}{\partial r^{2}}+\frac{1}{r} \frac{\partial w_{0}}{\partial r}+\frac{g^{2}}{r} \frac{\partial^{2} w_{0}}{\partial \varphi^{2}}=0, \quad(r, \varphi) \in A
$$

In Eq. (8)

$$
g=\sqrt{\frac{G_{\varphi z}}{G_{r z}}}
$$

The applied axial surface load on the mantle of cylindrical body (Fig. 2) is

$$
p_{z}=\frac{f}{2} \sin 2 \varphi, \quad r=R, \quad 0 \leq \varphi \leq 2 \pi, \quad 0 \leq z \leq L .
$$

Here $L$ is the length of the cylindrical body. It is very easy to show that the applied axial surface load is an equilibrium force system since

$$
\begin{gathered}
F_{x}=\int_{0}^{L} \int_{0}^{2 \pi} p_{z} R \mathrm{~d} \varphi \mathrm{d} z=\frac{f L R}{2} \int_{0}^{2 \pi} \sin 2 \varphi \mathrm{d} \varphi=0, \\
M_{x}=\int_{0}^{L} \int_{0}^{2 \pi} y p_{z} R \mathrm{~d} \varphi \mathrm{d} z=\frac{f L R^{2}}{2} \int_{0}^{2 \pi} \sin 2 \varphi \sin \varphi \mathrm{d} \varphi=0, \\
M_{y}=-\int_{0}^{L} \int_{0}^{2 \pi} x p_{z} R \mathrm{~d} \varphi \mathrm{d} z=-\frac{f L R^{2}}{2} \int_{0}^{2 \pi} \sin 2 \varphi \cos \varphi \mathrm{d} \varphi=0 .
\end{gathered}
$$

The traction boundary condition on the boundary surface can be written in the form

$$
G_{r z} \frac{\partial w_{0}}{\partial r}=\frac{f}{2} \sin 2 \varphi, \quad r=R, \quad 0 \leq \varphi \leq 2 \pi, \quad 0 \leq z \leq L .
$$

We look for the solution of the boundary value problem formulated by Eqs. (8) and (14) as

$$
w_{0}(r, \varphi)=F_{0}(r) \sin 2 \varphi .
$$

A simple computation gives the next result

$$
w_{0}(r, \varphi)=\frac{f R}{4 \sqrt{G_{r z} G_{\varphi z}}}\left(\frac{r}{R}\right)^{2 g} \sin 2 \varphi .
$$

Combination of Eqs. (5), (6), (16) yields the formulae of shearing stresses 
International Journal of Engineering and Management Sciences (IJEMS) Vol. 5. (2020). No. 2

DOI: 10.21791/IJEMS.2020.2.5.

$$
\begin{gathered}
\tau_{0 r z}=\frac{f}{2}\left(\frac{r}{R}\right)^{2 g-1} \sin 2 \varphi, \\
\tau_{0 \varphi z}=\frac{f}{2} g\left(\frac{r}{R}\right)^{2 g-1} \cos 2 \varphi .
\end{gathered}
$$

Cross-sectional shear forces $V_{0 x}, V_{0 y}$ and torsional moment $T_{0}$ can be computed from the next equations

$$
\begin{gathered}
V_{0 x}=\int_{A} \tau_{0 x z} \mathrm{~d} A, \quad V_{0 y}=\int_{A} \tau_{0 y z} \mathrm{~d} A \\
T_{0}=\int_{0}^{2 \pi} \int_{0}^{R} \tau_{0 \varphi z} r^{2} \mathrm{~d} r \mathrm{~d} \varphi
\end{gathered}
$$

In the present case

$$
\begin{gathered}
\tau_{0 x z}=\tau_{0 r z} \cos \varphi-\tau_{0 \varphi z} \sin \varphi, \\
\tau_{0 y z}=\tau_{0 r z} \sin \varphi-\tau_{0 \varphi z} \cos \varphi .
\end{gathered}
$$

Substitution Eqs. (17), (18) and Eqs. (21), (22) into Eqs. (19) leads to the following results

$$
V_{0 x}=0, \quad V_{0 y}=0 .
$$

Combination of Eq. (18) with Eq. (20) gives

$$
T_{0}=0
$$

Eqs. (23), (24) mean that the stress resultants acting on the cross section of cylindrical body vanish, so that the stresses $\tau_{0 r z}$ and $\tau_{0 \varphi z}$ form an equilibrium force system.

\section{Host cylinder with inclusion}

The material of the inclusion is isotropic, homogeneous linearly elastic, its shear modulus is denoted by $G$. The connection of the host body and inclusion is perfect. The centreline of the inclusion coincident with axis $z$ as shown in Fig. 2. The cross section of inclusion is the solid circular domain $A_{1}$ whose boundary curve is a circle with radius $a$ (Fig. 2). The strain state of inclusion is also antiplane shear deformation and its axial displacement is denoted by $w_{1}=w_{1}(r, \varphi)$. The shear strains and shear stresses in inclusion can be expressed as in terms of $w_{1}=w_{1}(r, \varphi)$

$$
\begin{gathered}
\gamma_{1 r Z}=\frac{\partial w_{1}}{\partial r}, \quad \gamma_{1 \varphi Z}=\frac{1}{r} \frac{\partial w_{1}}{\partial \varphi}, \\
\tau_{1 r Z}=G \frac{\partial w_{1}}{\partial r}, \quad \tau_{1 \varphi Z}=\frac{G}{r} \frac{\partial w_{1}}{\partial \varphi} .
\end{gathered}
$$

For the inclusion the equation of mechanical equilibrium can be represented as

$$
\frac{\partial^{2} w_{1}}{\partial r^{2}}+\frac{1}{r} \frac{\partial w_{1}}{\partial r}+\frac{1}{r^{2}} \frac{\partial^{2} w_{1}}{\partial \varphi^{2}}=0, \quad(r, \varphi) \in A_{1}
$$

Since the connection between the host body and inclusion is perfect we have

$$
w_{1}(a, \varphi)=\frac{f R}{4 \sqrt{G_{r z} G_{\varphi z}}}\left(\frac{a}{R}\right)^{2 g} \sin 2 \varphi, \quad 0 \leq \varphi \leq 2 \pi .
$$


We look for the solution of partial differential equation (27) according to Eq. (28) as

$$
w_{1}(r, \varphi)=F_{1}(r) \sin 2 \varphi, \quad(r, \varphi) \in A_{1} \cup \partial A_{1} .
$$

Substitution of Eq. (29) into Eq. (27) gives the following ordinary differential equation for $F_{1}=F_{1}(r)$

$$
\frac{\mathrm{d}^{2} F_{1}}{\mathrm{~d} r^{2}}+\frac{1}{r} \frac{\mathrm{d} F_{1}}{\mathrm{~d} r}-\frac{4}{r^{2}} F_{1}=0, \quad 0 \leq r \leq a .
$$

The bounded solution of Eq. (30) at $r=0$ can be written in the next form

$$
F_{1}(r)=C_{1} r^{2}, \quad 0 \leq r \leq a,
$$

that is

$$
w_{1}(r, \varphi)=C_{1} r^{2} \sin 2 \varphi
$$

In Eqs. (31) and (32) $C_{1}$ is an arbitrary constant, its value is obtained from the continuity condition of axial displacement on curve $\partial A_{1}$. A simple computation gives

$$
C_{1}=\frac{f R}{4 a^{2} \sqrt{G_{r z} G_{\varphi z}}}\left(\frac{a}{R}\right)^{2 g} .
$$

The continuity condition of normal traction on the curve $\partial A_{1}$ can be formulated in the following equation

$$
\tau_{0 r z}(a, \varphi)=\tau_{1 r z}(a, \varphi) .
$$

Shearing stress in radial direction in inclusion is given by the following equation

$$
\tau_{0 r z}(r, \varphi)=2 G C_{1} r \sin 2 \varphi .
$$

Combination of Eq. (34) with Eq. (35) gives

$$
\frac{f}{2}\left(\frac{a}{R}\right)^{2 g-1}=2 C_{1} G a .
$$

From Eqs. (33) and (36) it follows that

$$
\frac{f}{2}\left(\frac{a}{R}\right)^{2 g-1}=\frac{f}{2} \frac{G}{\sqrt{G_{r z} G_{\varphi z}}}\left(\frac{a}{R}\right)^{2 g-1}
$$

Eq. (37) is valid only if

$$
G=\sqrt{G_{r Z} G_{\varphi z}},
$$

so that there is a specific shear modulus $G$ of the inclusion for which the displacement field in the host body remains undisturbed and continuous in the compound cylinder consisting of host cylinder and inclusion cylinder. Benveniste and Chen [4] studied the problem of neutral inhomogeneity in the case of Saint-Venant torsion of nonhomogeneous circular bar. The material of the host bar is isotropic linear elastic with shear modulus $G$, and the inclusion is made of circular anisotropic bar with shear moduli $G_{r z}$ and $G_{\varphi z}$. The existence of neutral inhomogeneity for Saint-Venant torsion if the inclusion consists of only one component is also given by Eq. (38).

The cross sectional shear forces $V_{x}$ and $V_{y}$ and torsional moment $T$ in the coupled system of host cylinder and inclusion vanish since we have 
International Journal of Engineering and Management Sciences (IJEMS) Vol. 5. (2020). No. 2

$$
\begin{gathered}
V_{x}=\int_{0}^{2 \pi} \int_{0}^{R}\left[\left(\tau_{1 r z}-\tau_{0 r z}\right) \cos \varphi-\left(\tau_{1 \varphi z}-\tau_{0 \varphi z}\right) \sin \varphi\right] r \mathrm{~d} r \mathrm{~d} \varphi=0, \\
V_{y}=\int_{0}^{2 \pi} \int_{0}^{R}\left[\left(\tau_{1 r z}-\tau_{0 r z}\right) \sin \varphi+\left(\tau_{1 \varphi z}-\tau_{0 \varphi z}\right) \cos \varphi\right] r \mathrm{~d} r \mathrm{~d} \varphi=0, \\
T=\int_{0}^{2 \pi} \int_{0}^{R}\left(\tau_{1 \varphi z}-\tau_{0 \varphi z}\right) r^{2} \mathrm{~d} r \mathrm{~d} \varphi=0 .
\end{gathered}
$$

\section{Numerical example}

A concrete example illustrates the validity of the obtained result. Following data are used in this example: $R=0.4 \mathrm{~m}, a=0.15 \mathrm{~m}, G_{\varphi z}=7.5 \times 10^{9} \mathrm{~Pa}, G_{r z}=3.5 \times 10^{10} \mathrm{~Pa}, G=\sqrt{G_{r z} G_{\varphi z}}=1.62019 \times$ $10^{10} \mathrm{~Pa}$. Fig. 3 shows the contour lines of the axial displacement

$$
w(r, \varphi)=(H(r)-H(r-a)) w_{1}(r, \varphi)+H(r-a) w_{0}(r, \varphi) .
$$

In Eq. (42) $H=H(r)$ is the Heaviside function. The graphs of $w=w(r, \varphi)$ as a function of $r$ are shown in Fig. 4. for some different values of $\varphi(0, \pi / 6, \pi / 5, \pi / 4,3 \pi / 4,4 \pi / 5,5 \pi / 6)$. The contour lines of shearing stress

$$
\tau_{r z}(r, \varphi)=(H(r)-H(r-a)) \tau_{1 r z}(r, \varphi)+H(r-a) \tau_{0 r z}(r, \varphi)
$$

are given by in Fig. 5. The graphs of $\tau_{r z}=\tau_{r z}(r, \varphi)$ as a function of $r$ are presented in Fig. 6 for some different values of $\varphi(0, \pi / 6, \pi / 5, \pi / 4,3 \pi / 4,4 \pi / 5,5 \pi / 6)$.

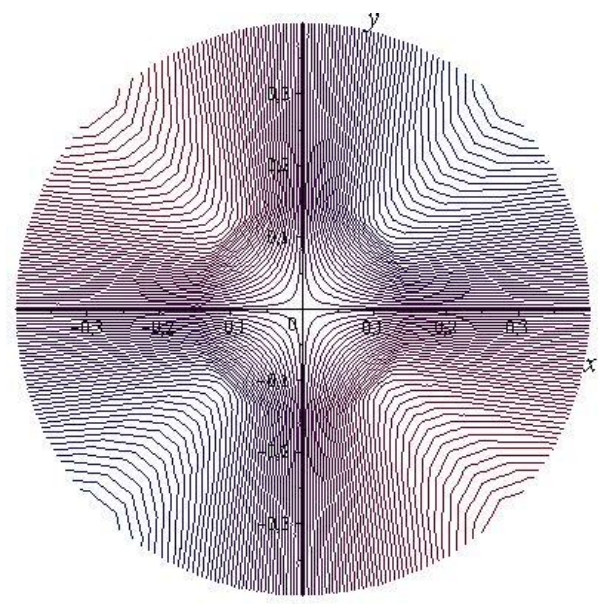

Figure 3. Contour lines of $w=w(r, \varphi)$. 
International Journal of Engineering and Management Sciences (IJEMS) Vol. 5. (2020). No. 2

DOI: 10.21791/IJEMS.2020.2.5.

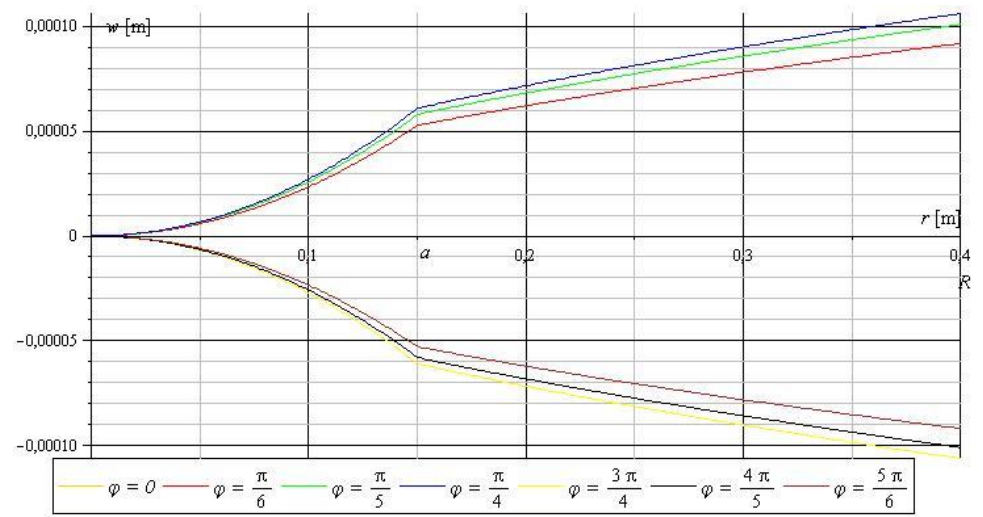

Figure 4. Plots of $w=w(r, \varphi=$ constant $)$.

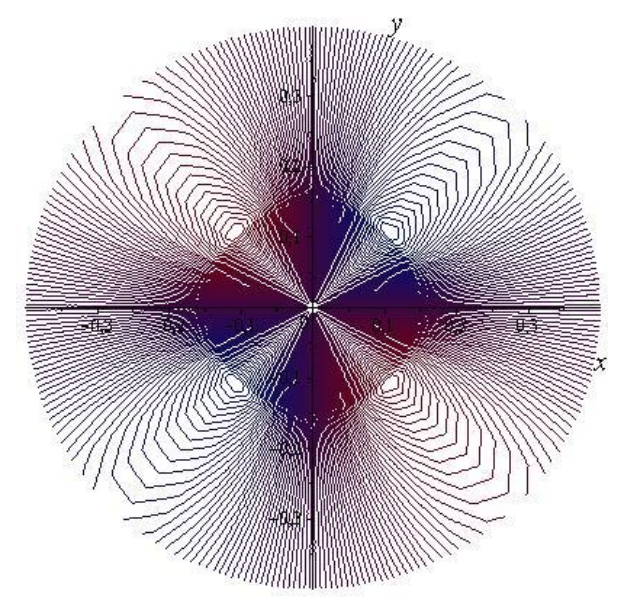

Figure 5. Contour lines of $\tau_{r z}(r, \varphi)$.

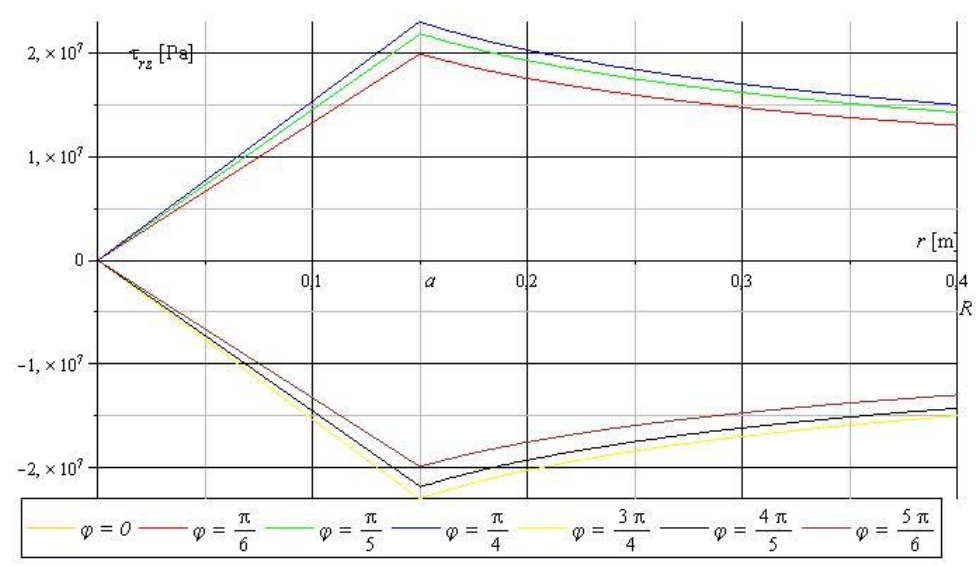

Figure 6. Plots of $\tau_{r z}(r, \varphi=$ constant $)$.

\section{Conclusion}

The problem of a single circular elastic inhomogeneity embedded within a cylindrical orthotropic cylinder whose curved boundary is loaded by surface traction acting on axial direction is investigated. The material of the embedded inhomogeneity is homogeneous isotropic, linearly elastic. The 
International Journal of Engineering and Management Sciences (IJEMS) Vol. 5. (2020). No. 2

DOI: 10.21791/IJEMS.2020.2.5.

deformation of the coupled host body and inclusion is antiplane shear deformation. Paper formulates the condition of existence of neutral inhomogeneity.

\section{Acknowledgement}

The described study was carried out as a part of the EFOP-3.6.1-16-2016-00011 "Younger and Renewing University - Innovative Knowledge City - institutional development of the University of Miskolc aiming at intelligent specialisation" project implemented in the framework of the Szechenyi 2020 program. The realization of this project is supported by the European Union, co-financed by the European Social Fund and supported by the National Research, Development and Innovation Office NKFIH, K115701.

\section{References}

[1] L. M. Milne-Thomson (1962), Antiplane Elastic Systems, Springer-Verlag, Berlin.

[2] T. C. T. Ting (1996), Anisotropic Elasticity. Theory and Application, Oxford University Press, Oxford.

[3] J. R. Barber (2010), Elasticity, Springer-Verlag, Berlin.

[4] Y. Benveniste, T. Chen (2003), The Saint-Venant torsion of a circular bar consisting of a composite cylinder assemblage with cylindrically orthotropic constituents, International Journal of Solids and Structures, 40(25), 7093-7107. 